Methods We conducted a register-based follow-up study of all gainfully employed 526839 men and 506092 women in Denmark $\left(56^{\circ} \mathrm{N}\right)$ born 1980 or later. We estimated annual mean occupational daytime (6:00-17:59 h) white light exposure levels (lux) for each year of employment 2000-2015 with a quantitative job exposure matrix (JEM). We followed participants from 2001 or first year of employment (if later) until first antidepressant treatment $\leq 2016$ as recorded in a national prescription database. We calculated incidence rate ratios adjusted for register information on age, calendar year, family history of antidepressant treatment, and educational level. Smoking was assessed with a lifestyle JEM.

Results During follow up, the population accumulated 6804 361 person-years and 30816 men (5.8\%) and 52817 women $(10.4 \%)$ initiated antidepressant treatment corresponding to 8 and 16 cases per 1000 person-years, respectively. Two percent of women and eight percent of men were exposed to an annual average above 500 lux. Among women, we observed reduced risk of initiating antidepressant treatment for those exposed the previous year to 500-999 lux (incidence rate ratio $[\mathrm{IRR}]=0.92,585$ cases, $95 \% \mathrm{CI} 0.85-1.00)$ and $\geq 1000$ lux (IRR $=0.90,294$ cases, 95\% CI 0.80-1.01) when compared with $<250$ lux. No association was apparent for men.

Conclusion Our results indicate a preventive effect of daytime light exposure on depression and other mental disorders treated with antidepressants for the small fraction of workers exposed to an annual mean above 500 lux at work, but only among women. Findings need replication in other countries.

\section{0-359 MENTAL HEALTH IN A SAMPLE OF 72,452 WORKERS FROM 8 COUNTRIES OF LATIN AMERICA: RESULTS OF HEALTH AND WORKING CONDITIONS SURVEYS}

${ }^{1}$ Paula Santia, Michael Silva-Peñaherrera, Fernando G Benavides. 'Universitat Pompeu Fabra, Spain

\subsection{6/OEM-2021-EPI.117}

Introduction Work is considered a key determinant of mental health. However, evidence on the impact of work in mental health in Latin America is scarce.

Objectives To analyse the relationship between mental health status and work in Latin America through three indicators: labour relationship (employed/self-employed or employer), employment condition (formal/informal), and occupational category (non manual skilled/non manual non-skilled/manual skilled/manual non-skilled).

Methods Cross sectional study based on health and working condition surveys from 8 countries of Latin America. Poor mental health was defined as a score of $>=3$ on the GHQ12; $>=10$ on the PHQ9; or $<=13$ on the WHO5 scales. Informal workers were defined as those unregistered or lacking a contract. Occupational categories were created following the international standard classification of occupation. Prevalence ratios and their IC95\% were calculated stratified by sex and adjusted by age, educational level and marital status.

Results Final sample consisted of 72,452 workers, from Argentina $(n=8966)$, Brazil $(n=52832)$, Costa Rica $(n=1503)$, El Salvador $\quad(n=1507), \quad$ Guatemala $(n=1510)$, Honduras $(n=1507)$, Nicaragua $(n=1500)$ and Panamá $(n=1505)$. Poor mental health ranged from $4.5 \%$ in Brazilian men to $25.9 \%$ in Panamanian women. Manual non-skilled men showed higher prevalence of poor mental health than non-manual skilled men in Argentina (RPa 1.43 IC95\% 1.04 - 1.95) and Central America (RPa 1.94 IC95\% 1.12 - 3.34). In Brazil, men employers showed higher prevalence of poor mental health (RPa 1.25 IC95\% 1.02 - 1.53) and informal workers showed higher prevalence of poor mental health irrespective of sex (Men RPa 1.16 IC95\% 1.02 - 1.33; Women RPa 1.30 IC95\% 1.05 - 1.60).

Conclusion Prevalence of poor mental health varies significantly between occupational groups and countries. Special attention should be pay to informal workers, workers in elementary occupations and employers.

\section{Radiation}

\section{0-138 CANCER MORTALITY THROUGH 2016 AMONG URANIUM MINERS ON THE COLORADO PLATEAU: INTERACTIONS OF RADON WITH SMOKING AND TIME SINCE EXPOSURE}

${ }^{1}$ Mary Schubauer-Berigan, Stephen Bertke, Kaitlin Kelly-Reif, Robert Daniels. 'International Agency for Research on Cancer, France

\subsection{6/OEM-2021-EPI.118}

Objective The long-term risks of lung cancer from radon progeny exposure are known largely from studies of uranium miners, including a cohort of 4137 male miners from the Colorado Plateau (CP) in the southwestern US. Our objective was to estimate the excess absolute risk (EAR) and excess relative risk (ERR) of lung and other cancer from radon exposure among CP uranium miners, evaluating the persistence of risk with 26 years of extended follow-up, and the form of interaction between radon and cigarette pack-years (PY).

Methods We followed the CP cohort for mortality through 2016, re-evaluated its radon exposure against original records, and imputed a smoking end date for cohort members. We evaluated EAR based on standardized rate ratios of lung cancer, adjusted for age, calendar year, and race. We modeled ERR using Cox proportional hazards regression of full risk sets (adjusted for age, race, birth year, exposure rate and time since last exposure). We also modeled variation in risk per unit exposure by attained age (AA) and time-since-exposure (TSE) and evaluated joint effects of radon and PY.

Results We identified 695 cases of lung cancer, including 146 among never- and light smokers. Lung cancer EAR per unit radon exposure increased substantially with smoking PY and throughout decades of follow-up. Lung cancer ERR decreased with AA and TSE, although to a lesser extent than observed elsewhere. The form of interaction between radon and PY was sub-multiplicative but greater-than-additive, appearing closer to multiplicative at lower radon exposures. Pancreas was the only other site showing a significantly positive ERR per unit exposure, although much lower than for lung cancer. Conclusion Excess risk of lung cancer persists throughout the lifespan among this cohort of uranium miners. Information about radon-smoking interactions, particularly at low exposure levels, is of interest for extrapolations to the general population. 